\title{
Phase Transition and Electrical Properties in $\mathrm{Cs}_{2} \mathrm{SeO}_{4 \cdot} \cdot \mathrm{Te}(\mathrm{OH})_{6}$
}

\author{
Litaiem H. ${ }^{1}$, Garcia-Granda S. $^{2}$, Dammak M${ }^{1}$., Mhiri T. ${ }^{3}$
}

1. Laboratoire de Chimie Inorganique, Faculté des Sciences de Sfax, Université de Sfax, B.P 1171 Sfax 3000 , Tunisie.

Email: litaiemh@yahoo.fr and meddammak@yahoo.fr

2. Laboratoire de Chimie Physique et Analytique, Faculté de Chimie, Université d'Oviedo, Oviedo 33006 , Espagne.

\section{Email : sgg@fq.uniovi.es}

3. Laboratoire physico-chimie de l'Etat Solide, Faculté des Sciences de Sfax, Université de Sfax, B.P 1171

\section{ABSTRACT} Sfax 3000, Tunisie.

\begin{abstract}
Dielectric investigations in the temperature and frequency $300-600 \mathrm{~K}$ and $0,1 \mathrm{KHz}-13 \mathrm{MHz}$, respectively, show that cesium selenate tellurate $\mathrm{Cs}_{2} \mathrm{SeO}_{4} \cdot \mathrm{Te}(\mathrm{OH})_{6}(\mathrm{CsSeTe})$ exhibits two phase transitions at 490 and $525 \mathrm{~K}$. The a.c. complex impedance measurements performed on CsSeTe material show an important level of conductivity at high temperature, attributed to the motion of $\mathrm{H}^{+}$proton. This behavior is in agreement with the presence of the super- protonic phase transition in CsSeTe compound at $525 \mathrm{~K}$. This assignment was confirmed by the analysis of the M"/M" ${ }_{\text {max }}$ Spectra. The temperature dependences of $\varepsilon_{r}^{\prime}$ and tan $\delta$ indicate that the anomaly at $490 \mathrm{~K}$ is attributed to a ferroelectric-paraelectric phase transition. Thermal analysis at high temperature, DSC, DTA, TG, Ms/z= 18 and Ms/z=32 confirm the presence of the two transitions already cited, the temperature and the nature of the decomposition.
\end{abstract}

Keywords: DSC; ATD; ATG; Dielectric properties.

\section{Council for Innovative Research}

Peer Review Research Publishing System

Journal: Journal of Advances in Chemistry

Vol. 7, No. 3

editor@cirworld.com

www.cirjac.com, member.cirworld.com 


\section{INTRODUCTION}

Telluric acid can form stable adducts with a wide range of organic and inorganic compounds of considerable importance as $\mathrm{Te}(\mathrm{OH})_{6}$ acts as both acceptor and donor of hydrogen bonds[1-7]. Some of those compounds, of general formula $\mathrm{M}_{2} \mathrm{AO}_{4} \mathrm{Te}(\mathrm{OH})_{6}$ (where $\mathrm{M}$ is monovalent cation and $\mathrm{A}=\mathrm{Se}$, S..), exhibit structural phase transition and interesting physical properties such as ferroelectricity and superionic-protonic conductivity [8-13].

X-ray crystallographic studies performed on a single crystal, showed that $\mathrm{Cs}_{2} \mathrm{SeO}_{4} \cdot \mathrm{Te}(\mathrm{OH})_{6}$ exhibits a monoclinic structure, at room temperature, with a centrosymmetric space group $\mathrm{P} 2{ }_{1} / \mathrm{c}$. The unit cell parameters are: $\mathrm{a}=12.817(6) \AA$, $b=7.519(3) \AA, c=12.432(5) \AA, \beta=111.16(2)^{\circ}$. In the CsSeTe structure, we note the presence of two different and independent anions in the same crystal. The structure in being built by different types of planes parallel on the bc one. In fact, planes containing the $\mathrm{Te}(\mathrm{OH})_{6}$ octahedra positioned at $\mathrm{x}=0$ and $\mathrm{x}=\mathrm{a} / 2$ and planes of $\mathrm{SeO}_{4}$ tetrahedra at $\mathrm{x}=\mathrm{a} / 4$. The $\mathrm{Cs}^{+}$cations are intercalated in bidimensional arrangement between the two types of planes. The Te atoms have an octahedral coordination. The Te-O distances are between 1.899(4) and 1.919(4) $\AA$ and the O-Te-O angles values vary from 87.6(2) and $92.4(2)^{\circ}$. The tetrahedral coordination of the Se atom is built with four oxygen atoms with Se-O distances varying from $1.630(4)$ and $1.649(4) \AA$. . In consequence, the O-Se-O angles are between $107.9(2)^{\circ}$ and $110.6(3)^{\circ}$. The $\mathrm{Cs}^{+}$ cations in the CsSeTe material are connected with ten to twelve oxygen atoms [10].

Both the selenate and the tellurate, in the CsSeTe structure, are connected with $\mathrm{O} . . \mathrm{H}-\mathrm{O}$ hydrogen bonds. The O...O distances are between 2.668(6) and 2.860(6) Á with O...H distances varying from 1.694(4) to 2.035(3) $\AA$. In consequence, the $\mathrm{O} . . . \mathrm{H}-\mathrm{O}$ angle values are between $133.8(3)$ and $172.2(3)^{\circ}$.

All these values of $\mathrm{O} \ldots \mathrm{H}$ and $\mathrm{O}-\mathrm{H} \ldots \mathrm{O}$ distances can favor the appearance of high protonic conductivity at high temperature which is the origin of the super-protonic phase transition characterized by the bricking of hydrogen bonds which link the anionic groups and the proton $\mathrm{H}^{+}$becomes free between the potential holes $\mathrm{SeO}_{4}^{2-}$ and $\mathrm{TeO}_{6}^{6-}[10]$.

The aim of the present work is to study the different phase transitions present in $\mathrm{Cs}_{2} \mathrm{SeO}_{4} \cdot \mathrm{H}_{6} \mathrm{TeO}_{6}(\mathrm{CsSeTe})$ compound. We report here the results obtained by, dielectric, differential scanning calorimetry (DSC), differential thermogravimetric analysis (TG), thermodifference analysis (D.T.A) and the associated mass spectrometry M/z 18 and 32 .

\section{EXPERIMENTAL PHASE}

Single crystals of $\mathrm{Cs}_{2} \mathrm{SeO}_{4} \cdot \mathrm{Te}(\mathrm{OH})_{6}$ were obtained by slow evaporation of an aqueous solution prepared by the addition, in stoichiometric quantities, of $\mathrm{Cs}_{2} \mathrm{CO}_{3}, \mathrm{H}_{2} \mathrm{SeO}_{4}$ and $\mathrm{H}_{6} \mathrm{TeO}_{6}$. The formula of the CsSeTe material were determined by chemical analysis and confirmed by structural refinement.

A Mettler-Toledo TGA/SDTA851 ${ }^{\mathrm{e}}$ and a DSC822 ${ }^{\mathrm{e}}$ were used for the thermal analyses in air atmosphere, at a heating rate of $10 \mathrm{~K} / \mathrm{min}$. 30.02 and $109.5 \mathrm{mg}$ of powder sample was thermally treated respectively in DSC and TG cases, and blank runs were performed.

In TG tests, a Pfeiffer Vacuum ThermoStar ${ }^{\text {TM }}$ GSD301 $\mathrm{T}$ mass spectrometer was used to determine the evacuated vapors. The masses $18\left(\mathrm{H}_{2} \mathrm{O}\right)$ and $32\left(\mathrm{O}_{2}\right)$ were tested by using a detector C-SEM, operating at $1200 \mathrm{~V}$, with a time constant of $1 \mathrm{~s}$.

The complex permittivity $\left(\varepsilon^{*}(\omega)=\varepsilon_{r}{ }^{\prime}-\mathrm{j} \varepsilon_{r}{ }^{\prime \prime}\right)$ was determined from electric capacitance measurements, the conductivity measurements were performed using a Hewlett-Packard 4192A LF automatic bridge monitored by a HP Vectra microcomputer in the range $0.1 \mathrm{KHz}-13 \mathrm{MHz}$. Temperature was measured with a chromel-alumel thermocouple close to the sample and data were collected in air in the temperature range $300-700 \mathrm{~K}$.

\section{RESULTS AND DISCUSSION}

\subsection{Calorimetric studies:}

The Differential Scanning Calorimetry trace shows the presence of three endothermic peaks. Heating process is shown in figure 1 . The first observed heat anomaly at $320 \mathrm{~K}$ clearly indicate a first order phase transition, The calculated transition enthalpy and entropy are $3.95 \mathrm{Jg}^{-1}$ and $1.2310^{-2} \mathrm{Jg}^{-1} \mathrm{~K}^{-1}$. An endothermic and intense peak at $530 \mathrm{~K}$ accompanied by two shoulders at $500 \mathrm{~K}$ and $545 \mathrm{~K}$ was observed, the enthalpy and entropy separately are respectively $196.82 \mathrm{Jg}^{-1}$ and $0.37 \mathrm{Jg}$ ${ }^{1} \mathrm{~K}^{-1}$. The anomaly at $726 \mathrm{~K}$ indicate a second order phase transition with the enthalpy and the entropy are $30.37 \mathrm{Jg}^{-1} \mathrm{and}$ $4.1810^{-2} \mathrm{Jg}^{-1} \mathrm{~K}^{-1}$.

The endothermic peak at $320 \mathrm{~K}$ can be attributed to the structural phase transition, which can favor a non centrosymmetric phase at high temperature $[12,14]$. 


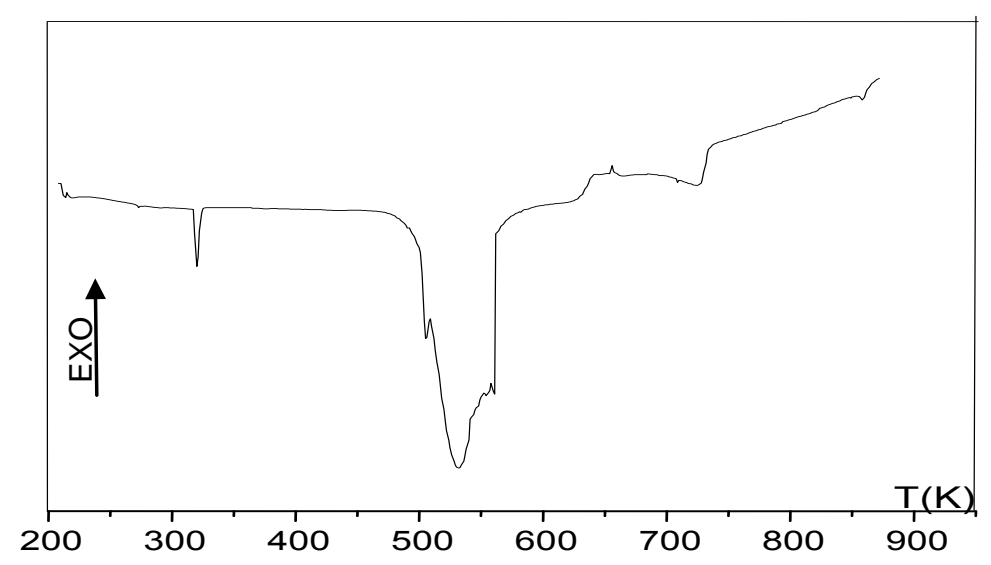

Fig 1: DSC heating curve of $\mathrm{Cs}_{2} \mathrm{SeO}_{4} \cdot \mathrm{Te}(\mathrm{OH})_{6}$

\subsection{Electric properties}

Polycrystalline pellets, $13 \mathrm{~mm}$ in diameter and $1.3 \mathrm{~mm}$ in thickness, were sintered at $400 \mathrm{~K}$ for $12 \mathrm{~h}$ under vacuum in order to eliminate the water content in the sample and obtaining dense pellet. We have undertaken an analysis of frequency response of electrical capacitance data relative to CsSeTe. This process is useful to determine the activation energies and conductivity relaxation time.

A combined analysis using both the complex modulus formalisms presents advantages over earlier methods. This theme has been futures developed in the present paper on CsSeTe. Our measurements were realized in the temperature range $300-700 \mathrm{~K}$. The complex modulus formalism $M^{*}=1 / \varepsilon^{*}=j \omega C_{0} Z^{*}$, where $j=\sqrt{-1}, \omega=2 \pi f$ is the angular frequency and $\mathrm{C}_{0}$ is the vacuum capacitance of the cell, has been adopted to determine the ion conductivity relaxation process. This formalism discriminates against electrode polarization and other interfacial effects in solid electrolytes [15].

Complex impedance spectra Z" versus Z' recorded at various temperatures are presented in Figure 2 . The complex impedance plane data show a non-depressed semi circle for all frequency and temperature variations. This behavior confirms that no electrode phenomenon is present. The resistance is determined by extrapolation at frequency equal to zero from the $Z^{\prime \prime}$ versus $Z^{\prime}$ circle arc centered under the $Z^{\prime}$ axis because at this frequency the equivalent circuit of our material presents only a resistant behavior.

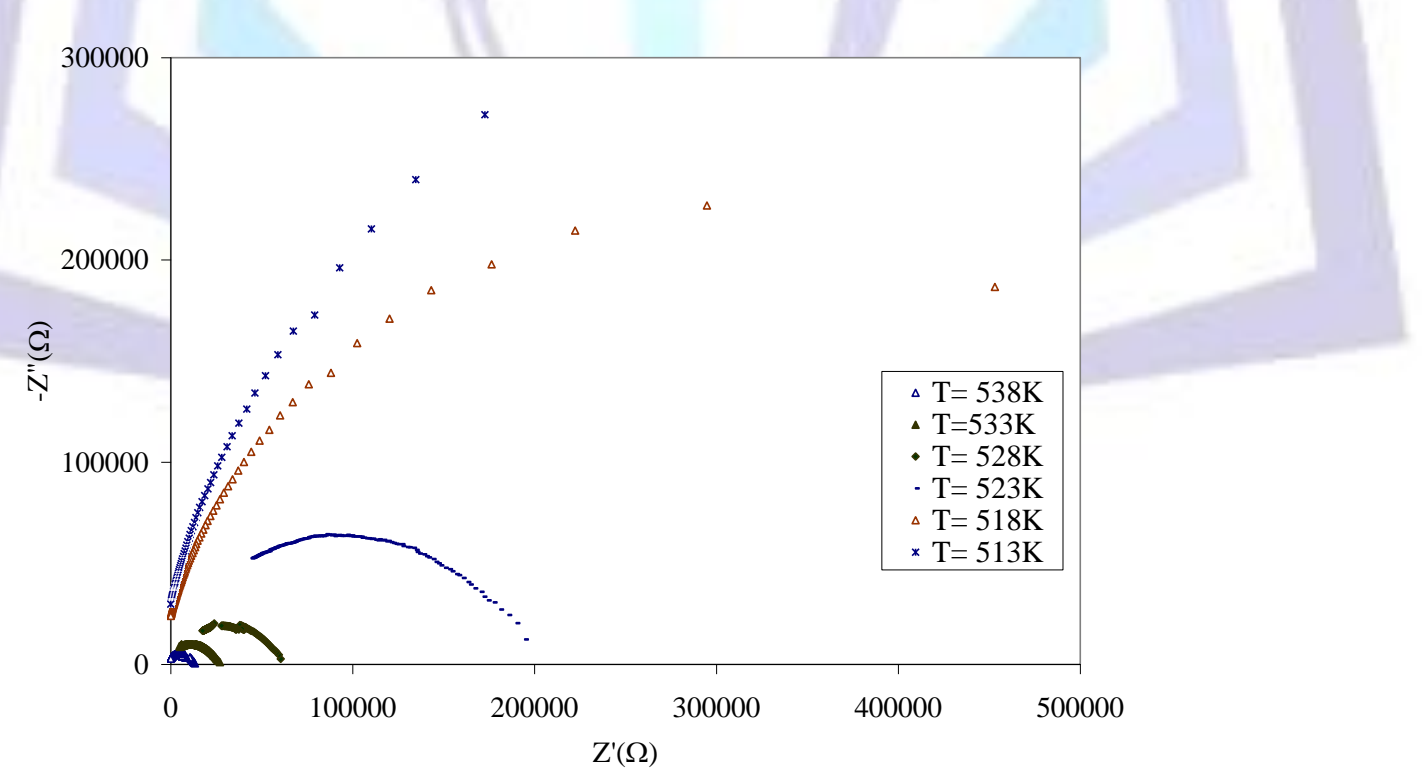

Fig 2: Complex impedance curves of $\mathrm{Cs}_{2} \mathrm{SeO}_{4} \cdot \mathrm{Te}(\mathrm{OH})_{6}$ at various temperatures.

The coefficient $\alpha$ which characterizes the deviation of the Cole-Cole from the Debye law is determined from the complex impedance spectrum $(\alpha=0.29)$ [16]. The small value of $\alpha$ can be considered as further confirmation that no electrode phenomenon is present. The curves of $Z$ " versus $Z$ ' show a rapid evolution of the resistance as a function of temperature. 
At about $525 \mathrm{~K}$, we observe a sudden decrease of the resistance. This temperature characterizes the breaking of hydrogen bonds.

Thermal evolution of conductivity for CsSeTe material is presented in figure 3.

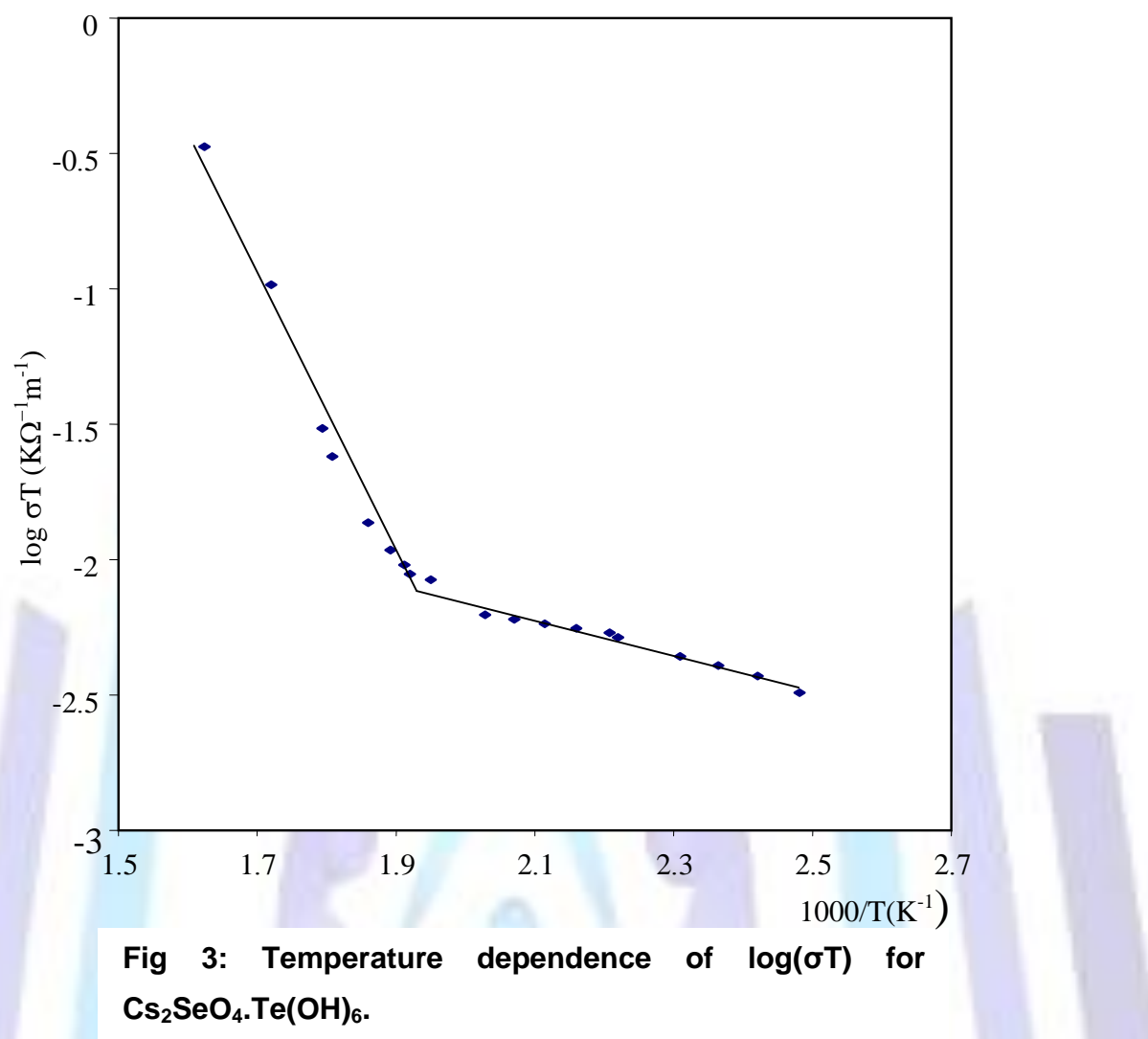

The plot in this figure shows the evolution of the conductivity $(\log (\sigma T)$ versus 1000/T) for CsSeTe compound. In the

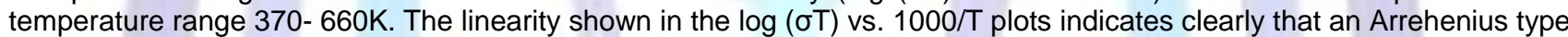
law $\left(\sigma \mathrm{T}=\sigma_{0} \exp (-\mathrm{Ea} / \mathrm{KT})\right)$ characterizes the low and the high temperature domain with a sudden jump increase of the conductivity up to $525 \mathrm{~K}$.

The conductivity plot exhibits two parts with a transition at about $480 \mathrm{~K}$, which can be attributed to the presence of ferroelectric-paraelectric phase transition.

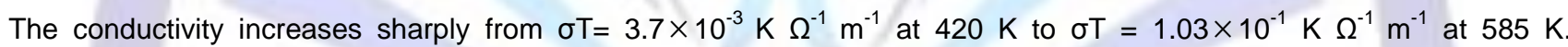
The activation energies at the conductor low temperature and the super-protonic conductor state are respectively $\Delta \mathrm{E}_{1}=$ $0.32 \mathrm{eV}$ and $\Delta \mathrm{E}_{2}=0.59 \mathrm{eV}$, this difference can be due to the difficulty of the proton displacement caused by the cell deformation introduced with the establishment of polar phase.

In spite of all the results indicated above, the high-temperature phase transition at about 525K in CsSeTe compound can be interpreted as a superionic-protonic one, observed previously at the same temperature by differential scanning calorimetry measurement. This phase transition can be due to the breaking of the $\mathrm{O}-\mathrm{H}$...O hydrogen bond which link oxygen atoms of the $\mathrm{SeO}_{4}$ tetrahedra to the hydroxide of $\mathrm{Te}(\mathrm{OH})_{6}$ octahedra.

In order to characterize the phase transition at about $500 \mathrm{~K}$ observed in DSC curve, we have studied the temperature dependence of the permittivity $\mathcal{E}_{r}{ }_{r}$ in the range $300-600 \mathrm{~K}$. Figure 4 shows the dielectric constant $\mathcal{E}_{r}$ evolution versus temperature at different frequency for CsSeTe material. From all those curves we observe that the permittivity increases and get a maximum value at $490 \mathrm{~K}$, this anomaly can be attributed to ferro-paraelectric phase transition [17, 19]. We observe that there is a significant variation of $\mathcal{E}_{r}$ with the frequency in this material. However, the real part of the permittivity can be considered as the sum of two contributions:

$\mathcal{E}_{r}^{\prime}=\mathcal{E}_{r}^{\prime}$ (latt.) $+\mathcal{E}_{r}^{\prime}$ (carr.), where $\mathcal{E}_{r}^{\prime}$ (latt.) and $\mathcal{E}_{r}^{\prime}$ (carr.) represent the lattice response due to permanent dipole orientation and the charge carrier response associated with long- range migration respectively [18]. The temperature of the maximum of the permittivity does not change with frequency i.e. there is no presence of relaxor type behavior in this frequency region. The intense peak in the permittivity observed at $\mathrm{T}=520 \mathrm{~K}$ is attributed to the superprotonic phase transition. This behavior is observed in the case of $\mathrm{Rb}_{2} \mathrm{SeO}_{4} \cdot \mathrm{Te}(\mathrm{OH})_{6}$ and $\left(\mathrm{NH}_{4}\right)_{2} \mathrm{SeO} \cdot \mathrm{Te}(\mathrm{OH})_{6}[12,17]$. 
More over the reasons given to attribute the ferroelectric character to this phase transition is not sufficient and we are convinced that only measuring the spontaneous polarization can confirm the presence of this character. However the important difficulty in realizing this manipulation is the small size of the crystal which is not appropriate for this measurement and the measurement realized on pellet introduce some secondary effect due to grain boundaries.

Figure 5 shows the evolution of the dissipation factor (Tanס) as function of temperature. The obtained values are relatively in agreement with the important contribution of the conductivity in this material. Tan $\delta$ increases from low temperature present a maximum below Tc then decreases and present a minimum in the vicinity of $490 \mathrm{~K}$. This behavior corroborates the presence of a ferroelectric-paraelectric phase transition in CsSeTe compound at Tc $=490 \mathrm{~K}$. In fact, the CsSeTe structure is being built up by planes of pure $\mathrm{SeO}_{4}^{2-}$ tetrahedra and planes of $\mathrm{TeO}_{6}^{6-}$ octahedra altering by the $\mathrm{Cs}^{+}$cations. This arrangement can explain that the presence of the ferro-paraelectric phase transition at $490 \mathrm{~K}$ due to the coexistence of two different charge center [18, 19].

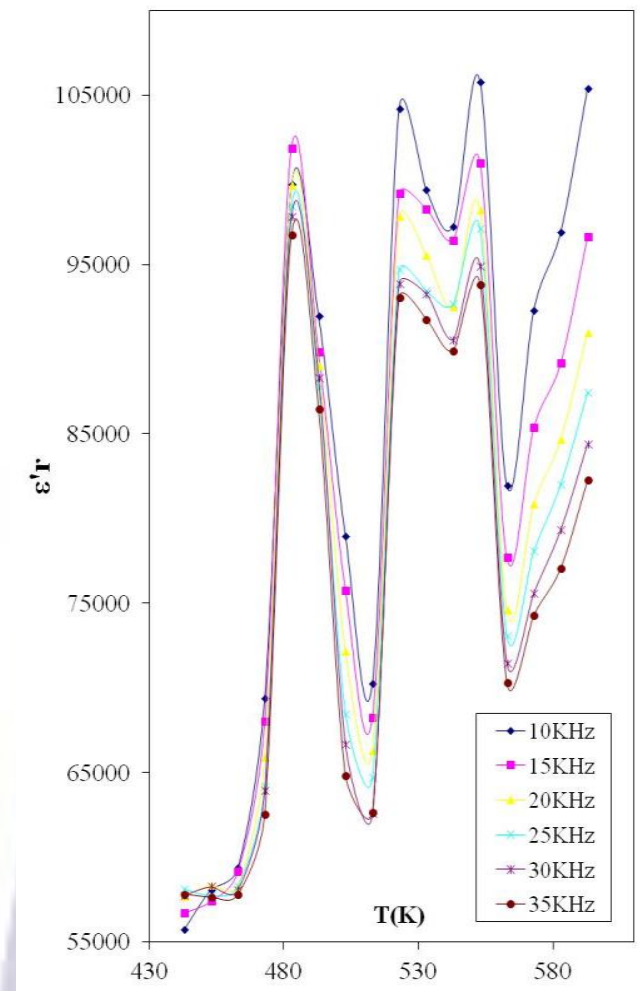

Fig 4: Temperature dependence of $\varepsilon_{r}^{\prime}$ as a function of frequency for $\mathrm{Cs}_{2} \mathrm{SeO}_{4} \cdot \mathrm{Te}(\mathrm{OH})_{6}$.

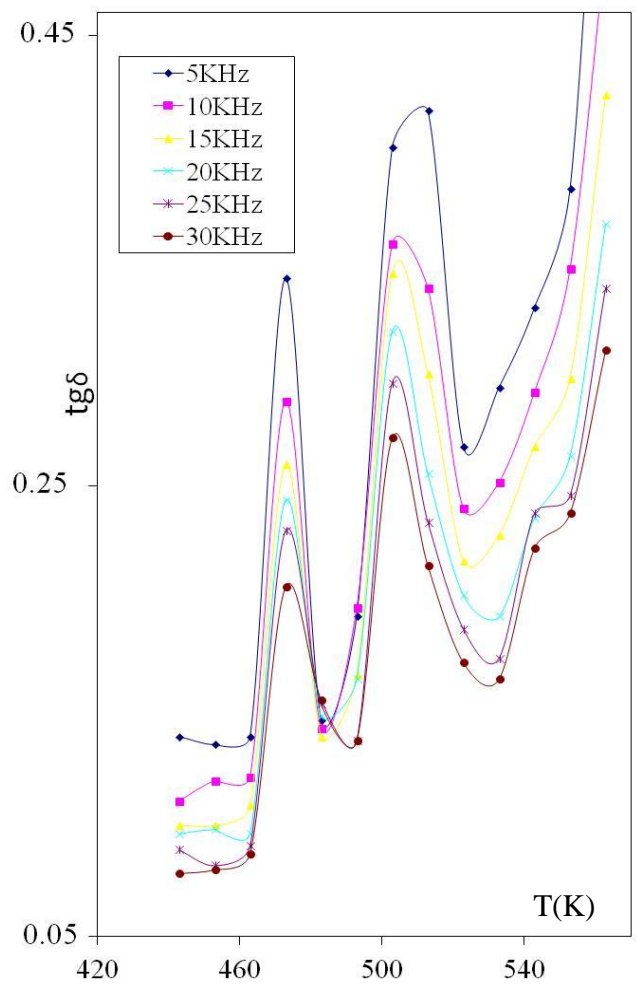

Fig 5: Thermal evolution of the dissipation factor as a function of frequency for $\mathrm{Cs}_{2} \mathrm{SeO}_{4} \cdot \mathrm{Te}(\mathrm{OH})_{6}$.

In order to know the conductivity mechanism we have extended our study to the complex modulus $\mathrm{M}^{*}$ formalism, the real part $\mathrm{M}^{\prime}$ and the imaginary part $\mathrm{M}^{\prime \prime}$ of the $\mathrm{M}^{*}$ complex modulus $\left(\mathrm{M}^{*}=\mathrm{M}^{\prime}+j \mathrm{M}^{\prime \prime}\right)$ were calculated from the complex impedance data $\left(Z^{*}=Z^{\prime}-j Z^{\prime \prime}\right)$ using the relations $M^{\prime}=\omega C_{0} Z^{\prime \prime}$ and $M^{\prime \prime}=\omega C_{0} Z^{\prime}$.

In figure 6 the calculated values of real part of the complex modulus show that at all temperature, the value of M' reaches a constant value $\left(\mathrm{M}_{\infty}^{\prime}=1 / \mathcal{E}_{\infty}\right)$ at high frequencies. At low frequencies, it approaches zero, which indicate that the electrode polarization phenomena make a negligible contribution to $\mathrm{M}^{*}$ and may be ignored when the electric data are analyzed in this form [20]. 


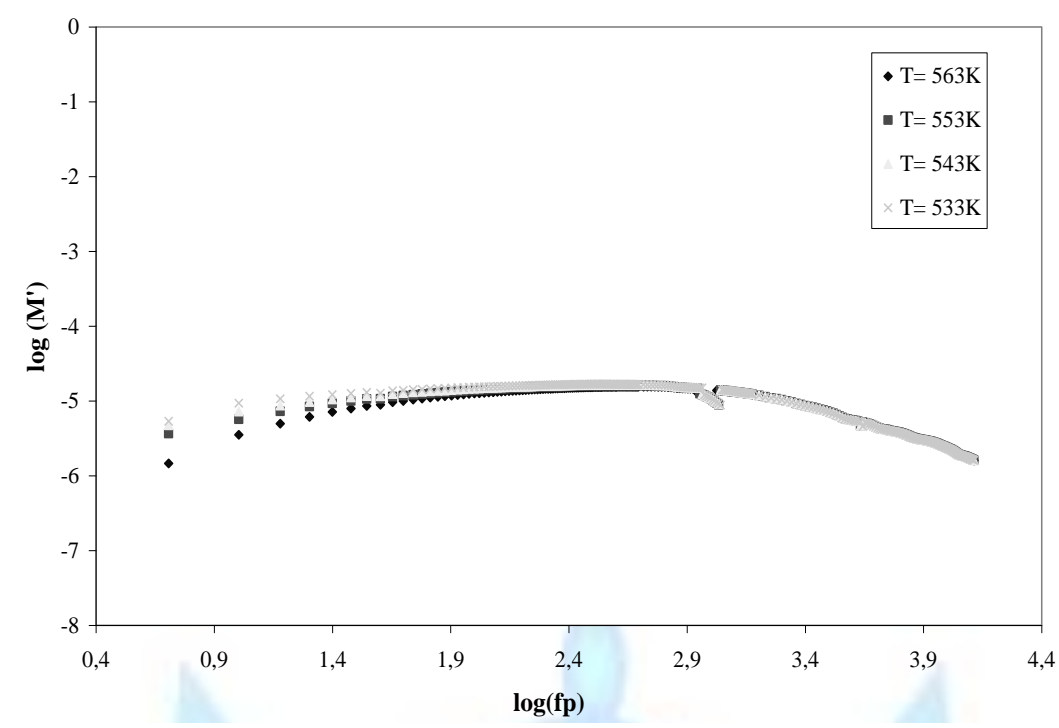

Fig 6: A plot of $\log M '$ versus $\log (f)$ at various temperature for $\mathrm{Cs}_{2} \mathrm{SeO}_{4} \cdot \mathrm{Te}(\mathrm{OH})_{6}$

The plots of the normalized $\mathrm{M}^{\prime \prime} / \mathrm{M}^{\prime \prime}{ }_{\max }$ imaginary part of the complex modulus versus $\log (\mathrm{f})$ are given in Figure 7 at various temperature for CsSeTe material. The $\mathrm{M}^{\prime \prime} / \mathrm{M}^{\prime \prime}$ max relative to high temperature given shows an non exponential behavior of the electrical function that is well described by the empirical stretched exponential function $\varphi(t)=\exp \left[\left(-\mathrm{t} / \tau_{\sigma}\right) \beta^{\beta}\right](0<$ $\beta<1)[21,22]$.
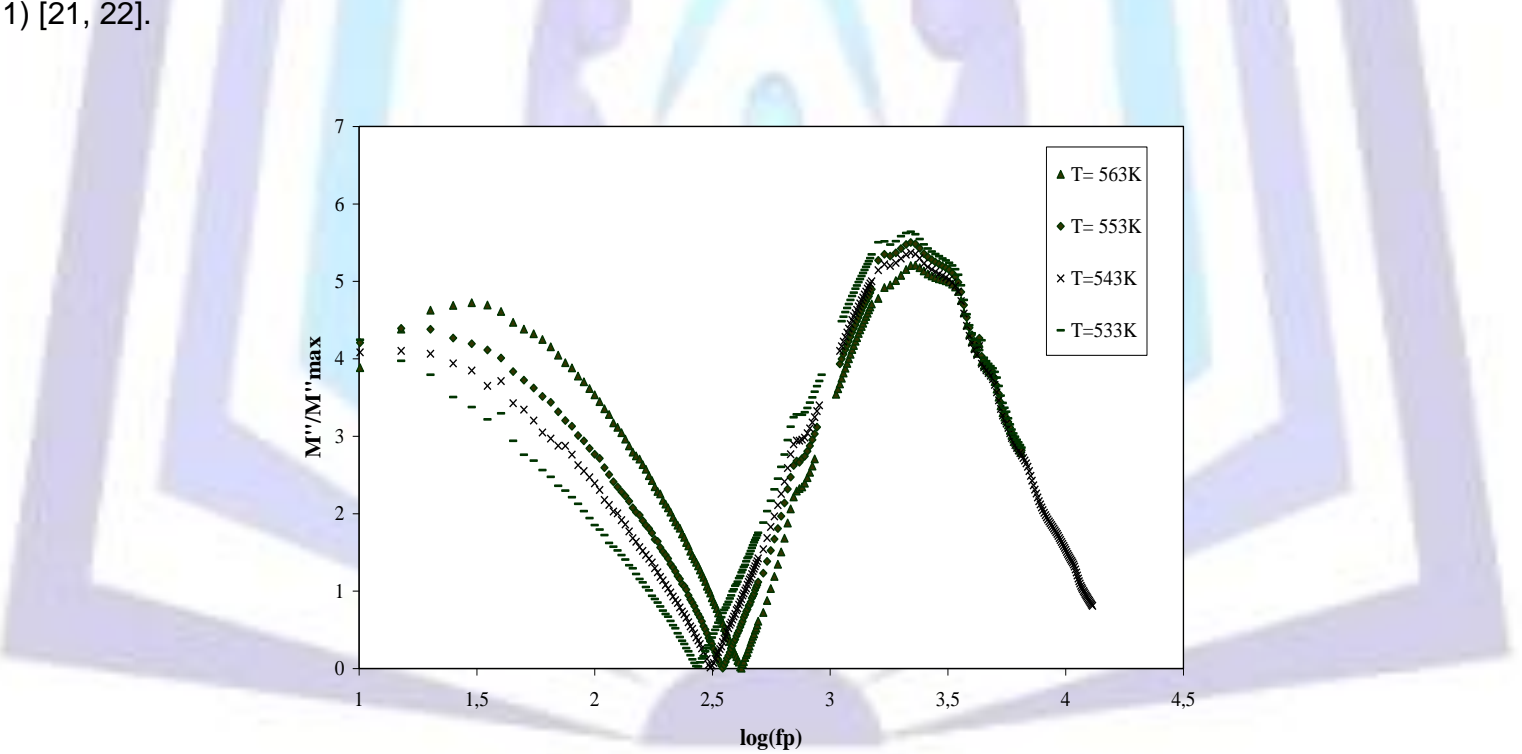

Fig 7: A plot of normalized modulus (M"/M" $\left.{ }_{\max }\right)$ versus $\log (f)$ at

various temperature for $\mathrm{Cs}_{2} \mathrm{SeO}_{4} \cdot \mathrm{Te}(\mathrm{OH})_{6}$.

Where $\tau_{\sigma}$ is the relaxation time, $\beta$ is the fractional Kohlrausch exponent which characterizes the shift from exponential behavior and $\varphi(\mathrm{t})$ is the Kohlrausch function. The region to the left of the peaks is where the $\mathrm{H}^{+}$ions are mobile over long distances; the region to the right is where the ions are spatially confined to their potential wells. The frequency range where the peak occurs is indicative of the transition from short-range to long-range mobility at decreasing frequency and is defined by the condition $\omega \tau_{\sigma}=1$ [23].

\subsection{Thermal behavior}

The thermal stability of CsSeTe was investigated. In fact, differential thermal analysis (DTA) and thermo gravimetric (TG) reveal that no mass loss was detected before $545 \mathrm{~K}$.

The TG/DTG, DTA curves, and the mass spectrometric analysis, are depicted in the figure 8 and 9. 
TG /DTG curves of CsSeTe reveal total mass losses of, respectively, 17.5\% (cal. 15.95\%), from 200 to $900 \mathrm{~K}$.

The mass loss of CsSeTe proceeds by four separate and defined steps. The first step between $510 \mathrm{~K}$ and $583 \mathrm{~K}$ (which reaches its maximum velocity at $543 \mathrm{~K}$ ), with the mass loss of $7.65 \%$ (cal. $8.45 \%$ ), associated with endothermic peak at $550 \mathrm{~K}$ and $547 \mathrm{~K}$ on the DTA and DSC curves respectively, corresponds to the loss of ( 3 moles) water molecules: resulting from the decomposition of telluric acid $\mathrm{Te}(\mathrm{OH})_{6}$ as follows:

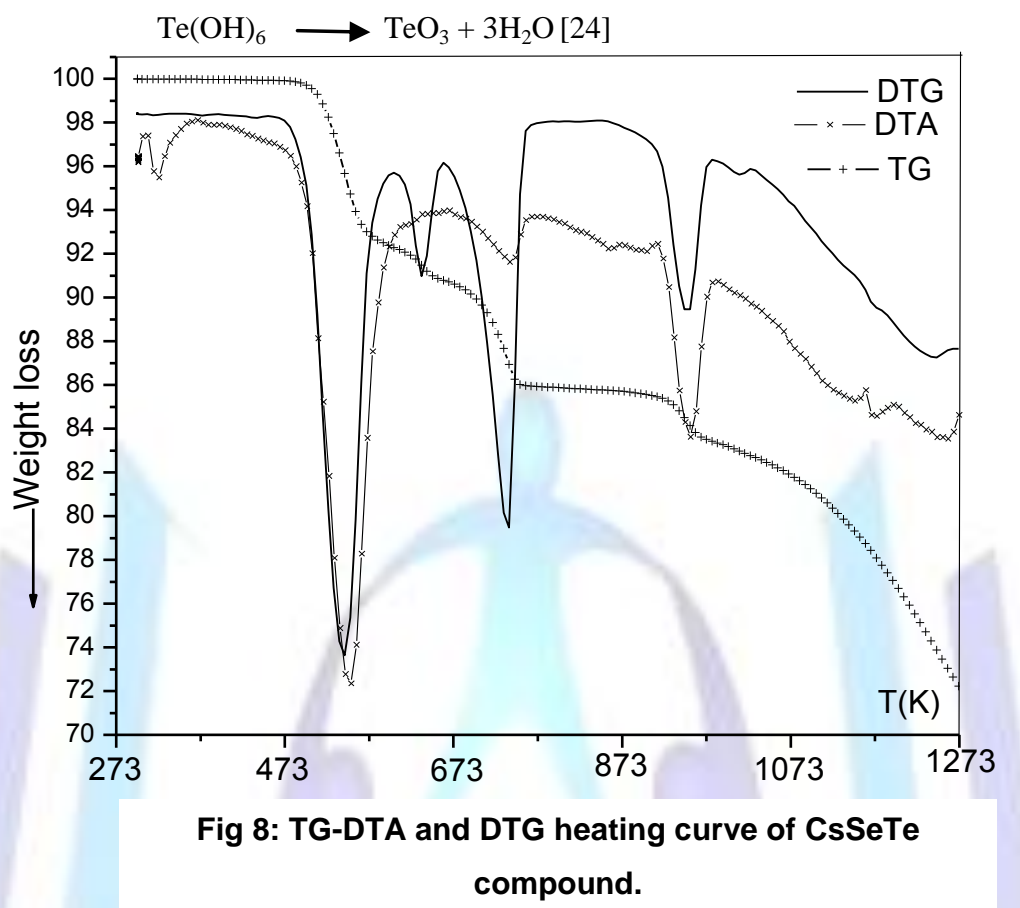

The second step, between $593-663 \mathrm{~K}$, with total mass loss of $1.62 \%$ (which reach its maximum velocity at $636 \mathrm{~K}$ ) and a main peak on the DSC at (630K), it can be considered as a continuation of the first loss by evaporation of $0.8 \%$ of the total mass to lead the liberation of $3 \mathrm{H}_{2} \mathrm{O}$ already described and the beginning of the third loss that is manifested by evaporation of $\mathrm{O}_{2}$ that occurs in two stages. In fact, the third step, between 663-753K, with total mass loss of $4.88 \%$ (cal $5 \%$ ) (Which reach its maximum velocity at $735 \mathrm{~K}$ ) and a main peak on the DTA (at 740K) and DSC (at 723K) curves. The fourth step, between $907-990 \mathrm{~K}$, with total mass loss of $2.85 \%$ (cal 2.5\%) (Which reach its maximum velocity at 953K) and a main peak on the DTA (at 955K) curves corresponding to the liberation of another oxygen atom.

The associated mass spectrometry M/z 18 and 32 curves are in a good agreement with TG/DTG data. M/z 18 curve has one maxima at $551 \mathrm{~K}$ (accompanied by a boss at $645 \mathrm{~K}$ ), corresponding to the loss of three water molecules. M/z $32 \mathrm{curve}$ presents three maximum respectively at 625,710 and $903 \mathrm{~K}$.

Oxygen atom is liberated in three waves, the first two waves correspond to oxygen loss with the formation of $\mathrm{Te}_{2} \mathrm{O}_{5}$ and the third wave corresponds to the formation of tellurium dioxide (tetragonal modification), which melts to yield amorphous tellurium dioxide and the formation of the solid product can be described by the

scheme:

$$
\mathrm{TeO}_{3} \rightarrow \mathrm{Te}_{2} \mathrm{O}_{5} \longrightarrow \mathrm{TeO}_{2}[24]
$$




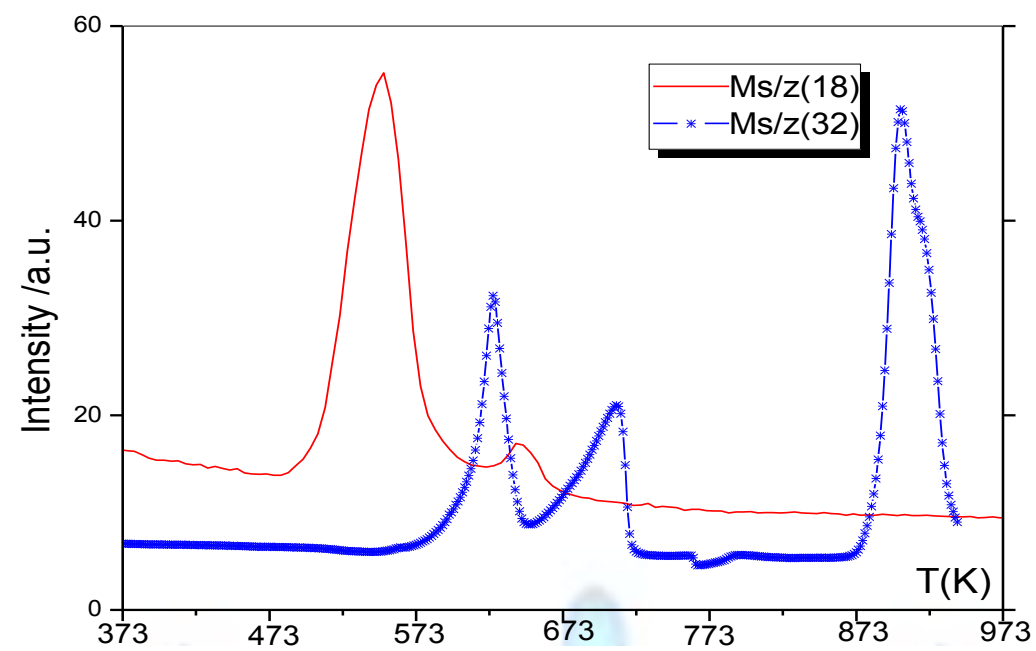

Fig 9: M/Z $18\left(\mathrm{H}_{2} \mathrm{O}\right)$ and $\mathrm{M} / \mathrm{z} 32\left(\mathrm{O}_{2}\right)$ MS signals of evacuated

\section{4- CONCLUSION}

vapors for CsSeTe material.

The coexistence of two independent and different anions, $\mathrm{TeO}_{6}^{6-}$ and $\mathrm{SeO}_{4}^{2-}$ in the same unit cell, connected by hydrogen bonds, can be the source of important physical properties such as the ferroelectricity and super-protonic conduction at high temperature. In fact, CsSeTe material presents, in one hand, a ferro-parelectric phase transition, at $490 \mathrm{~K}$, detected by DSC and confirmed by dielectric constant and the dissipation factor versus temperature. On the other hand, we remark a strong jump detected at $525 \mathrm{~K}$ in the conductivity plot characterizes a super-protonic conduction accompanied by the breaking of hydrogen bonds which link all the polyhedra in our material structure. As a result, the proton moves between the potential wells associated with the anionic and cationic entities. Thermal behavior reveals that no mass loss was detected before $545 \mathrm{~K}$.

\section{ACKNOWLEGMENT}

This work is supported by the minister of superior education and research of Tunisia. All the authors express their thanks to $\mathrm{Dr}$ N. Zouari for his help in the dielectric measurement and for his fruitful discussions.

\section{REFERENCES}

[1] Dammak, M., Cousson. A. and Nierlich, M., Acta Cryst E61 (2005) i55.

[2] Litaiem, H., Dammak, M., Mhiri, T. and Cousson, A., J. Alloys and compounds, 396 (2005) 34.

[3] Bechibani, I., Litaiem, H., Ktari, L., Lhoste, J. and Dammak, M., J. Molucular structure, 1045 (2013) 199.

[4] Zilber, R., Durif, A. and Averbuch-Pouchot, M.T., Acta Cryst. B36 (1980)2743.

[5] Zilber, R., Torjman, I. and Guitel, J.C., Acta Cryst. B36 (1980) 2741.

[6] Zilber, R., Durif, A. and Averbuch-Pouchot, M.T., Acta Cryst. B37 (1981)650.

[7] Zilber, R., Durif, A. and Averbuch-Pouchot, M.T., Acta Cryst. B38 (1982)1554.

[8] L. Ktari, Dammak, M., Kolsi, A.W. and Cousson, A., J. Alloys and Compounds 476 (2009) 54.

[9] Dammak, M., Ktari, L., Cousson, A. and Mhiri, T., J. Solid State Chemistry 178 (2005) 2109.

[10] Dammak, M., Mhiri, T., Jaud, J., Savariault, J.M., J. Inorganic Materials 3 (2001) 861.

[11] Dammak, M., Litaiem. H., Mhiri, T. and Daoud, A.,J. Alloys Compd. 442 (2007) 316.

[12] Litaiem. H., Dammak, M., Mhiri, T., Cousson, A., J. Alloys Compd. 396 (2005) 34.

[13] Dammak, M., Litaiem. H., Mhiri, T., J. Alloys Compd. 416 (2006) 228.

[14] Ktari, L., Dammak, M., Mhiri, T. and Savariault, J.M., J. Solid State Chemistry 161 (2001) 1.

[15] Howell, F. S.,. Bose, R. A, Mace, P. B., Moynihan, C. T., J. Phys. Chem. 78 (1987) 53.

[16] cole, K. S., cole, R. H., J. Chem. Phys. 43 (1941) 341. 
[17] Litaiem. H., Dammak, M., Mhiri, T., J. Phy. Chem. News 46 (2009) 48.

[18] Khemakhem, H., Ferroelectrics 231 (1997) 47.

[19] Dammak, M., Khemakhem, H., Zouari, N., Mhiri, T., Kolsi, A.W., J. Solid State lonics 127 (2000) 125.

[20] Williams, C., Watts, D. C., Trans. Farady Soc. 23 (1970) 625.

[21] Ngai, K. L., Martin, S. W., Phys. Rev. B40 (1989) 10550.

[22] Patel, H. K., Martin, S. W., Phys. Rev. B45 (1992) 10292.

[23] Chowdari, B. V. R., Gopalakrishnan, R., J. Solid State Ionics 69 (1994) 53.

[24] Faby, J., Loub, J. and Feltl, L., J. Thermal analysis 24 (1982) 95. 